\title{
Progress toward an ambulatory pump-lung
}

\author{
Zhongjun J. Wu, PhD, ${ }^{a}$ Mark Gartner, $\mathrm{MS}^{\mathrm{b}}{ }^{\mathrm{b}}$ Kenneth N. Litwak, PhD, DVM, ${ }^{\mathrm{c}}$ and Bartley P. Griffith, $\mathrm{MD}^{\mathrm{a}}$
}

From the Department of Surgery, Univer-

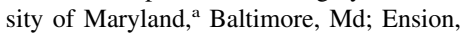
Inc, ${ }^{\text {b }}$ Pittsburgh, Pa; and the Department of Surgery, University of Louisville, ${ }^{c}$ Louisville, Ky.

This study was partially supported by a National Institutes of Health grant (R01HL 65740) and carried out while the authors were with the University of Pittsburgh.

Received for publication Feb 17, 2005; revisions received April 13, 2005; accepted for publication April 22, 2005.

Address for reprints: Bartley P. Griffith, MD, Department of Surgery, University of Maryland Baltimore, N4W94, $22 \mathrm{~S}$ Greene St, Baltimore, MD 21201 (E-mail: bgriffith@ smail.umaryland.edu).

J Thorac Cardiovasc Surg 2005;130:973-8

$0022-5223 / \$ 30.00$

Copyright $\odot 2005$ by The American Association for Thoracic Surgery

doi:10.1016/j.jtcvs.2005.04.032
Objectives: Currently available therapies for acute and chronic lung diseases have not been effective and have various problems associated with the technologies used. We present a novel active mixing pump-lung with the goal of providing total respiratory support to ambulatory patients.

Methods: The pump-lung is based on the concept of active mixing oxygenation within a constrained vortex. The rotation of hollow-fiber membranes disrupts the concentration boundary layer, increasing gas exchange efficiency, and simultaneously pumps the blood. Consequently, the amount of membranes required to achieve gas transfer sufficient for total respiratory support is considerably small. A series of studies, including computational design, experimental bench testing, and in vivo animal experiments, have been performed to implement this concept into a viable artificial pump-lung device.

Results: A series of pump-lung prototypes with a membrane surface area of 0.17 to $0.5 \mathrm{~m}^{2}$ were designed and characterized in vitro with bovine blood, demonstrating extremely high gas exchange efficiency. The prototype with a gas exchange surface area of $0.5 \mathrm{~m}^{2}$ was evaluated in calves. The device provided oxygen transfer of approximately $115 \mathrm{~mL} / \mathrm{min}$ for respiratory support of an animal for up to 5 days.

Conclusions: Progress to date suggests a high likelihood of success for an extracorporeal shorter-term lung that can be switched in and out like dialysis devices. Our device is unique in that it incorporates an integrated pumping and active mixing principle for excellent gas transfer and eliminates the need of the native right ventricle's ability to power blood through the artificial and natural lungs.

$\mathrm{O}$ ur group has been interested in long-term cardiopulmonary support since 1978, when we initiated our clinical program of extracorporeal membrane oxygenation (ECMO) for children and began a laboratory-based investigation of novel methods and devices for the task. With the gradual acceptance of lung transplantation for end-stage pulmonary disease, we noted an unacceptable attrition of patients waiting for scarce organs. We participated in advances made in mechanical circulatory support for bridge to transplantation and destination therapy for heart failure and hoped for a similar option for our patients with lung disease. Our approach to the problem blended our knowledge of hollow-fiber membrane gas transfer and of blood pumps. ${ }^{1,2}$ On the basis of our large experience with candidates for lung transplantation, unlike other passively perfused devices, ${ }^{1,2}$ we reasoned that in many patients right ventricular function would unreliably pump blood through an oxygenator, and we virtually planned to fuse a blood pump on a large-fiber oxygenator. We explored the possibility of combining active mixing and pumping function in a compact unit. At present, there is an overall reduced cardiac surgical market for perfusion and little corporate support for the development of an artificial lung. We were fortunate to pursue our interest through the National Heart, Lung, and Blood Institute (HL 065740). We set the following criteria as the drivers for the design: (1) oxygen $\left(\mathrm{O}_{2}\right)$ delivery rate of $250 \mathrm{~mL} / \mathrm{min}$ and carbon dioxide $\left(\mathrm{CO}_{2}\right)$ elimination rate of $200 \mathrm{~mL} / \mathrm{min}$; (2) blood pumping of $5 \mathrm{~L} / \mathrm{min}$; (3) minimized 

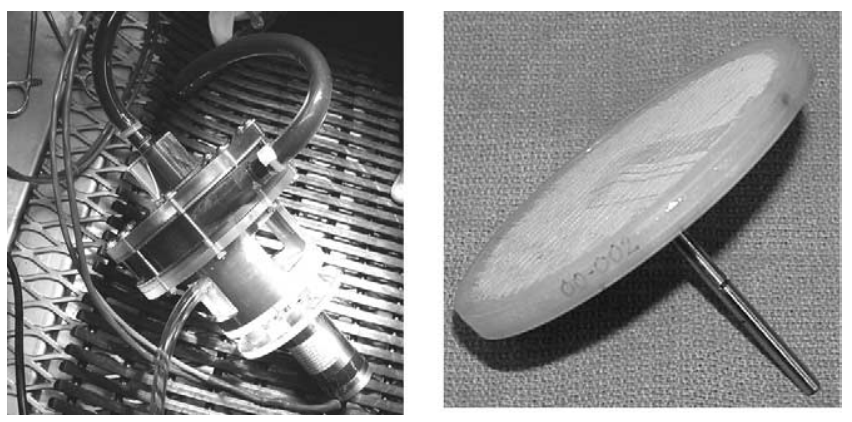

Figure 1. Active mixing pump-lung (APL) device.

surface area for gas exchange $\left(0.5 \mathrm{~m}^{2}\right)$; (4) low bloodpriming volume $(<100 \mathrm{~mL})$; (5) minimal blood cell injury (hemolysis and platelet loss or activation); (6) anticoagulation with warfarin; and (7) support for at least 21 days.

This report presents the results of the first 2 years of our supported effort toward an ambulatory pump-lung and frames the remaining hurdles that we face in this difficult but approachable field.

\section{Materials and Methods Device Description}

The ambulatory pump-lung (APL) is based on the active convective mixing and pumping concept, which was achieved by rotating a disk of microporous hollow-fiber membranes (Figure 1). A duallumen shaft was used to supply gas through a spoke to the half of the periphery of the disk and exit the waste gas from the other half of the periphery. While the gas travels through the lumens of the hollow fibers from one side of the disk to the other, gas exchange occurs as the blood flows across the hollow-fiber membrane surfaces. The waste gas is collected on the opposite side of the disk, where it then returns to the shaft for exhaust through another spoke. The disk is housed in a specially designed housing with a single axial inlet, primarily cylindrical volute, and single radial outlet. When rotated in the housing, the centrifugal force caused by the rotation of the disk draws the blood over the fibers and propels it radially. The disk is rotated by using an electric DC motor coupled to the disk shaft through a lip seal. The flow paths of the supply gas and exhaust gas are also separated with lip seals. A series of APL prototypes with 4-inch-diameter disks of gas exchange surface areas ranging from 0.17 to $0.5 \mathrm{~m}^{2}$ and with inlet and outlet diameters of 0.5 inch were constructed with polypropylene microporous hollow-fiber membranes. The priming volume of the complete APL device is about $100 \mathrm{~mL}$.

\section{Fluid Dynamics Analysis}

Our APL device required detailed understanding of the fluid regimen to allow the geometric manipulations necessary to optimize the relative velocity between fibers and blood. Computational fluid dynamics (CFD) was leveraged to enhance understanding of these key flow features. A general-purpose CFD code (CFX version 5.6; Ansys Inc, Pittsburgh, Pa) was used to model the fluid flow within the APL device.
In parallel, experimental flow visualization was also carried out with laser-based fluorescent particle imaging. ${ }^{3}$ Neutrally buoyant fluorescent microspheres $30 \mu \mathrm{m}$ in diameter (Duke Scientific, Palo Alto, Calif) were seeded into a water-glycerin blood analog fluid as markers of flow. The fluid had a viscosity of about $3.7 \mathrm{cP}$ and a density of $1.08 \mathrm{~g} / \mathrm{cm}^{3}$ at room temperature (approximately $20^{\circ} \mathrm{C}$ ) to match the rheologic property of blood at high shear rates. The flow fields at the region of interest were imaged with a highresolution digital CCD camera (Megplus 1.4; Eastman Kodak, San Diego, Calif) with a digital frame grabber board (Oculus-F/64; Coreco, Inc, St-Laurent, Quebec, Canada) hosted in a personal computer under various operating conditions.

\section{In Vitro Evaluation of APL}

A closed circulatory loop with heparinized bovine blood (hemoglobin, $12 \pm 1 \mathrm{~g} / \mathrm{dL}$ ) was used to evaluate the gas transfer performance of the APL prototypes. The inlet blood was conditioned with a Medtronic Maxima oxygenator to an oxygen saturation of $65 \% \pm 5 \%$, a temperature of $37^{\circ} \mathrm{C} \pm 2{ }^{\circ} \mathrm{C}$, a $\mathrm{PCO}_{2}$ of 45 $\pm 5 \mathrm{~mm} \mathrm{Hg}$, and a base excess of $0 \pm 5 \mathrm{mEq} / \mathrm{L}$. The condition of the inlet blood is consistent with the Association for the Advancement of Medical Instrumentation standard for testing blood oxygenators. ${ }^{4}$

Three samples of inlet and outlet blood were taken at each of several device rotation rates and the gas flow/blood flow ratio was set at 1:1. The partial pressures of $\mathrm{O}_{2}$ and $\mathrm{CO}_{2}$ were analyzed with a blood gas analyzer (ABL5; Radiometer, Brønshøj, Denmark). The concentration of hemoglobin and $\mathrm{O}_{2}$ saturation of the blood at the inlet and outlet of the APL device were measured with a co-oximeter (OSM3, Radiometer). The blood flow rate and pressure head generated by the APL device were monitored by using the transonic flow meter (T101; Transonic System Inc Ithaca, NY) and physiologic pressure transducers (Model PX272; Edwards Lifesciences, Irvine, Calif) with a patient monitor (Spacelabs, Hillsboro, Ore). The oxygen gas flow rate was measured with a gas flowmeter (822 Top-Trak; Sierra Instruments, Monterey, Calif). $\mathrm{CO}_{2}$ concentration in the exhaust gas was determined by using a $\mathrm{CO}_{2}$ analyzer (CO2-44B; Physio-Dyne, Quogue, NY). The oxygen transfer rate was calculated by using the Fick principle with the above measured quantities. The $\mathrm{CO}_{2}$ transfer was calculated on the basis of the exit gas flow rate and the concentration of $\mathrm{CO}_{2}$.

A similar in vitro circulatory loop consisting of the APL device and a temperature-controlled reservoir was constructed to evaluate the hemolytic performance and biocompatibility. The heparinized bovine blood was circulated for up to 6 hours. The pressures at the inlet and outlet ports were measured by using the physiologic pressure transducers with the patient monitor. The blood flow rate was measured with the ultrasonic flowmeter. Interval blood samples during the experimental run were taken for complete blood counts (platelets, white blood cells [WBCs], and hematocrit), as well as plasma free hemoglobin (PFH). The complete blood count of the blood samples was measured with a hematology analyzer (Antech Diagnostics, Lake Success, NY). PFH was measured with spectrophotometry. ${ }^{5}$

The pumping performance of the APL device was evaluated over the full operating range of flow from shutoff to stall at 4 rotational speeds in the above circulatory flow loop. A series of 


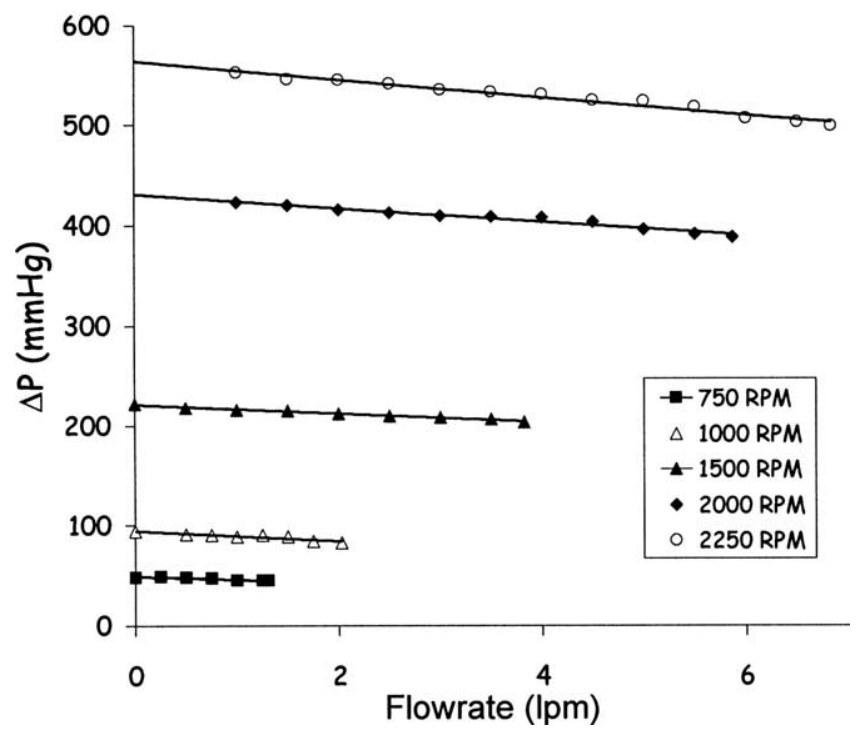

Figure 2. Pumping function of the active mixing pump-lung device as a blood pump.

pressure increase versus flow curves were collected to characterize the pumping capability of the APL device.

\section{In Vivo Evaluation}

The APL was surgically connected between the right atrium (RA) and the right carotid artery of Jersey calves. During the course of the study, all animals received humane care in accordance with the "Guide for Care and Use of Laboratory Animals" (National Institutes of Health publication no. 86-23, revised 1996) and the approved protocol by the Institutional Animal Care and Use Committee of the University of Pittsburgh.

A Thoratec ventricular assist device atrial cannula with a Teflon collar was placed in the RA via the right jugular vein and secured with double purse-string sutures. A Thoratec ventricular assist device 14-mm-diameter arterial cannula was anastomosed end to side to the right carotid artery. Both cannulas were extended to the upper right lateral chest wall using biocompatible tubing and then connected to the APL mounted on the animal's back. Anticoagulation was initiated with a loading dose of heparin to produce activated clotting times of greater than 400 seconds (calf normal value, approximately 150 seconds). After surgical intervention, a slow infusion of heparin was begun, with the intention of maintaining the activated clotting time at approximately 250 seconds. Warfarin $(10 \mathrm{mg})$ was started the evening after the operation and periodically adjusted to maintain an international normalized ratio of between 2.5 and 3.5. The heparin infusion was discontinued thereafter.

Transonic flow probes were placed on the outlet cannulas. Oxygen saturation was continuously monitored by using optical oximetry probes attached to the inlet and outlet cannulas. Blood pressure increase across the APL unit and animal arterial and pulmonary artery (PA) pressures were continuously monitored with the physiologic pressure transducers with the patient monitor.

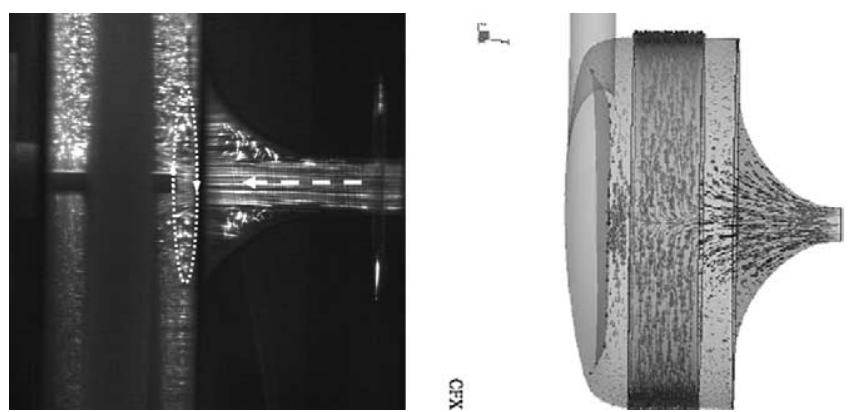

Figure 3. A, Typical flow image of the active mixing pump-lung device operated at $1000 \mathrm{rpm}$ to generate a flow rate of $3 \mathrm{~L} / \mathrm{min}$ against $92 \mathrm{~mm} \mathrm{Hg}$. B, CFD-predicted flow velocity field within the active mixing pump-lung device.

The blood compatibility of the APL was monitored in terms of WBC and platelet counts, as well as PFH generation. Each animal underwent a complete necropsy examination at the study termination. Special attention was paid to the device and the animal's lungs and pulmonary vasculature. Pulmonary vessels were searched for the presence of emboli or abnormalities.

\section{Results}

\section{In Vitro Pumping Function}

As a blood pump, the ability of the APL device to pump the blood is presented in Figure 2. This particular prototype had a membrane surface area of $0.17 \mathrm{~m}^{2}$. The pumping function is expressed as the device-generated pressure versus flow curves at a specific rotation speed. The pressure versus flow curves exhibited a relatively flat trend with increase of flow rate over a wide range at each rotational speed. This feature indicates that the APL had a wide operating range of flow rate against a large physiologic pressure load.

\section{Flow Characteristics}

The APL device exhibited a unique flow characteristic. The rotation of the hollow fibers generated a unique constrained vortex in the space between the disk and APL upper housing (Figure 3,A). This vortex encircled the axial inlet flow, which entered the porous hollow fibers and was radially spun out of the hollow-fiber bed to enter the abovementioned vortex. A similar vortex was also formed in the space between the disk and APL lower housing, but part of the fluid exited at the outflow tract. It was observed that the portion of the constrained vortices recirculated back to the hollow-fiber bed for further mixing. These unique flow features specific to the rotating disk of fabric membranes were observed under all operating conditions.

The CFD-predicted flow features with blood as the working fluid are consistent with the experimentally observed flow patterns. Typical fluid velocity field at the APL mid- 

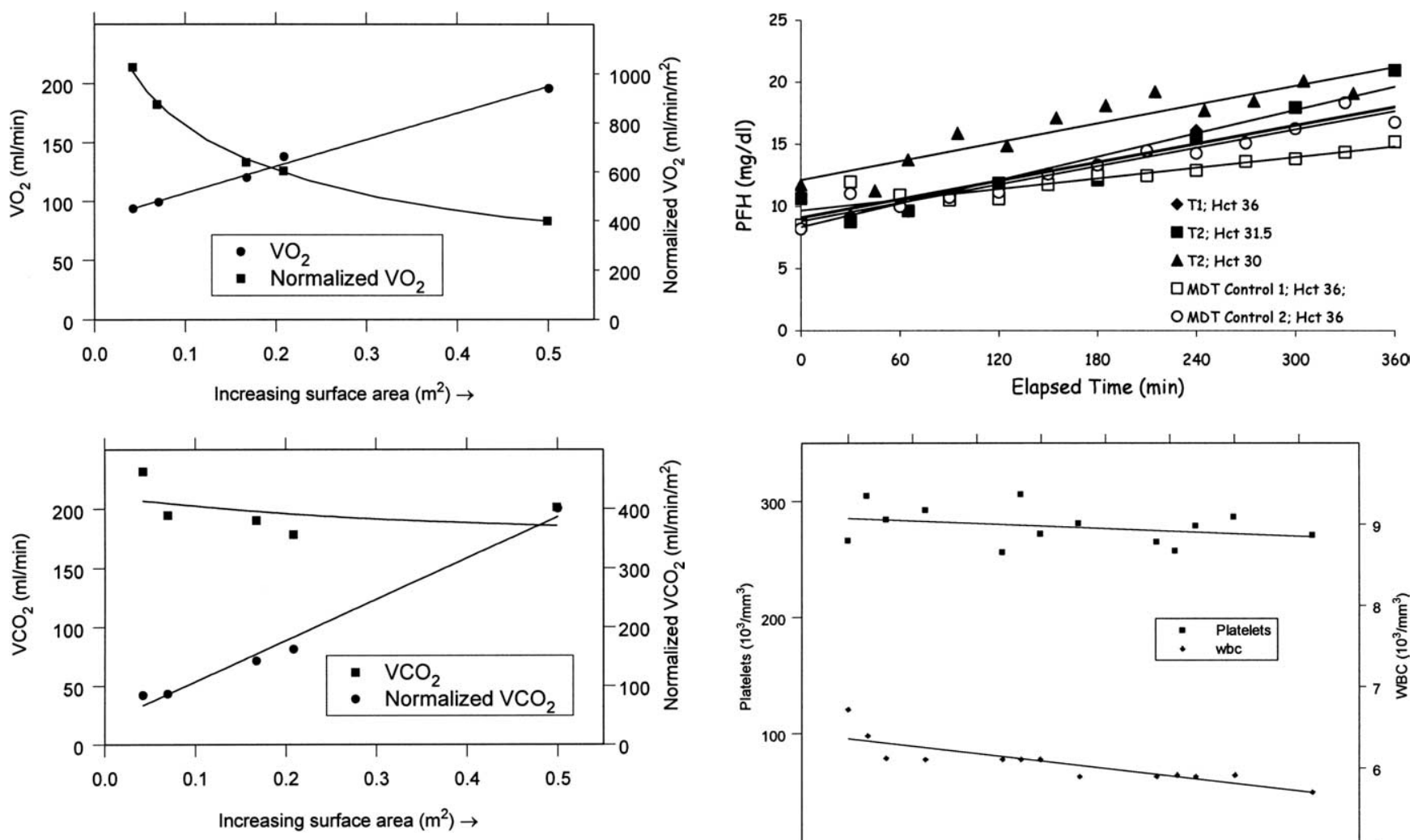

Figure 4. Gas exchange performance of the active mixing pumplung device versus surface area of membrane (Association for the Advancement of Medical Instrumentation test conditions: $\mathbf{5}$ L/min; normalized index of hemolysis, $0.068 \mathrm{~g} / 100 \mathrm{~L}$ ). $\mathrm{VO}_{2}, 0 x y g e n$ transfer rate; $\mathrm{VCO}_{2}$, carbon dioxide transfer rate.

plane, as well as flow streamlines, are shown in Figure $3, B$. The CFD model of the APL was comprised of multiple domains representing the porous rotor and the surrounding fluid.

\section{In Vitro Gas Transfer}

Both the $\mathrm{O}_{2}$ and $\mathrm{CO}_{2}$ transfer rates of the APL prototypes with a range of surface area are presented in Figure 4. With a surface area of $0.5 \mathrm{~m}^{2}$, the APL device was capable of delivering gas transfer rates of approximately $200 \mathrm{~mL} / \mathrm{min}$ for $\mathrm{O}_{2}$ and $200 \mathrm{~mL} / \mathrm{min}$ for $\mathrm{CO}_{2}$ at a blood flow rate of 5 $\mathrm{L} / \mathrm{min}$. Although the normalized gas transfer rates decreased with increase of the surface area from 0.17 to $0.5 \mathrm{~m}^{2}$, our APL device with a surface area of $0.5 \mathrm{~m}^{2}$ still exhibited extremely high gas transfer rates of $400 \mathrm{~mL} \cdot \mathrm{min}^{-1} \cdot \mathrm{m}^{-2}$ for $\mathrm{O}_{2}$ and $370 \mathrm{~mL} \cdot \mathrm{min}^{-1} \cdot \mathrm{m}^{-2}$ for $\mathrm{CO}_{2}$.

\section{In Vitro Biocompatibility}

The result of the hemolysis testing for a $0.17-\mathrm{m}^{2}$ APL device at a rated flow of $3.5 \mathrm{~L} / \mathrm{min}$ is presented in Figure 5, $A$. The 6-hour hemolysis experiments indicated that the

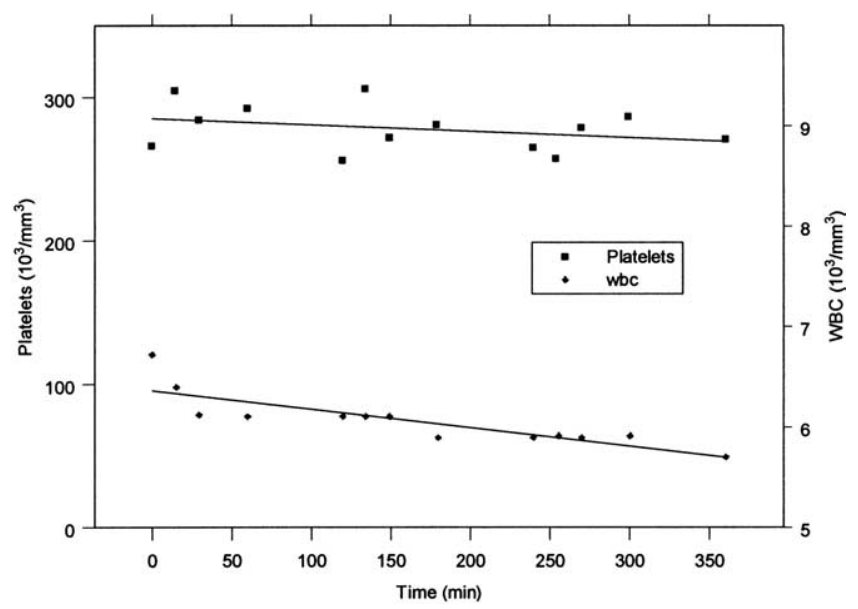

Figure 5. A, Results of the hemolysis testing over 6 hours on the bench in accordance with the Association for the Advancement of Medical Instrumentation standards at a rated flow of $3.5 \mathrm{~L} / \mathrm{min}$. $B$, Platelet and white blood cell (WBC) counts decreased modestly over the 6-hour testing of the active mixing pump-lung device in vitro with bovine blood. MDT, Medtronic Forte oxygenator/roller pump; $P F H$, plasma free hemoglobin; Hct, hematocrit.

APL device exhibited hemolytic characteristics on par with those of a control system consisting of a roller pump and a Medtronic Forte membrane oxygenator. The platelet and WBC counts over 6 hours of in vitro testing are presented in Figure 5, $B$. The platelet and WBC counts are within the normal measurement error margins for both measurements. It was also observed that no thrombus deposition was found within the device during posttest examination. When the surface areas were scaled up, the normalized index of hemolysis from a $0.5-\mathrm{m}^{2}$ APL prototype was $0.068 \mathrm{~g} / 100 \mathrm{~L}$, which is in the range of the currently used blood pumps ${ }^{6}$ and commercial blood oxygenators. ${ }^{7}$

\section{In Vivo Evaluation}

In vivo animal experiments with the implantable APL device were conducted in 5 calves. The implantable APL device used both uncoated and silicone-coated hollow-fiber 
membranes with a surface area of $0.5 \mathrm{~m}^{2}$. The mean blood flow rate was approximately $4 \mathrm{~L} / \mathrm{min}$. The sweep gas flow rate was maintained at 1:1. All animals tolerated the surgical implantation and recovered. During the 1-day in vivo bovine experiments, the implanted APL device with uncoated hollow-fiber membranes delivered a normalized oxygen transfer rate of $350 \mathrm{~mL} \cdot \mathrm{min}^{-1} \cdot \mathrm{m}^{-2}(175 \mathrm{~mL} / \mathrm{min})$, which was close to the in vitro result. One APL prototype with silicone-coated fiber membranes was used for a 5-day in vivo study. The oxygen gas transfer from the 5 -day study is shown in Figure 6. A temporary and unexplained decrease in oxygen transfer occurred at 40 hours but was considered most likely caused by change in animal position and kinking of inlet cannulas. The mean oxygen transfer rate was approximately $110 \mathrm{~mL} / \mathrm{min}$. The silicone-coated hollow-fiber membranes seemed to reduce the average gas transfer rate modestly ( $175 \mathrm{~mL} / \mathrm{min}$ to $110 \mathrm{~mL} / \mathrm{min})$ in this configuration. $\mathrm{PFH}$ was increased from a preoperative level of $5 \mathrm{mg} / \mathrm{dL}$ to 28 $\mathrm{mg} / \mathrm{dL}$ postoperatively and remained increased over the period of 5 days. The platelet and WBC counts remained in the normal physiologic ranges. Necropsy results exhibited that there were no infarcts in the heart, lung, and kidney.

\section{Discussion}

Lung disease is America's third largest cause of death. ${ }^{8}$ Adult respiratory distress syndrome afflicts approximately 150,000 patients every year in the United States, ${ }^{9}$ and despite advances in critical care, mortality remains between $30 \%$ and $70 \% .^{10}$ Currently available advanced therapies for patients with chronic respiratory failure include mechanical ventilation and ECMO. With mechanical ventilation, often the tidal volume, airway pressure, and oxygen fraction necessary to achieve sufficient gas exchange can further damage the lungs ${ }^{11}$ including barotrauma, volutrauma, and other iatrogenic injuries. ECMO systems are associated with several major problems: complexity of operation, bleeding caused by the need for high levels of anticoagulation, and limited mobility of the patient. Recent studies reported the novel use of arteriovenous pumpless oxygenation that can benefit some with acute lung failure, but this method has only been used to date in the intensive care unit setting, and flow is limited. ${ }^{12,13}$

Although lung transplantation has become an effective treatment over the past 20 years, fewer than 1000 lung transplantations are performed in the United States annually because of the shortage of organ donors. Approximately $13 \%$ of patients listed for lung transplantation die while waiting for scarce donor organs. ${ }^{14}$ Unlike heart and kidney transplantation candidates, patients with irreversible acute and chronic lung disease have no bridge option and must live poor-quality lives, often terminally bound to a mechanical ventilator and rarely receiving high-risk, last-option transplantations. Implantable artificial lungs are always thought to
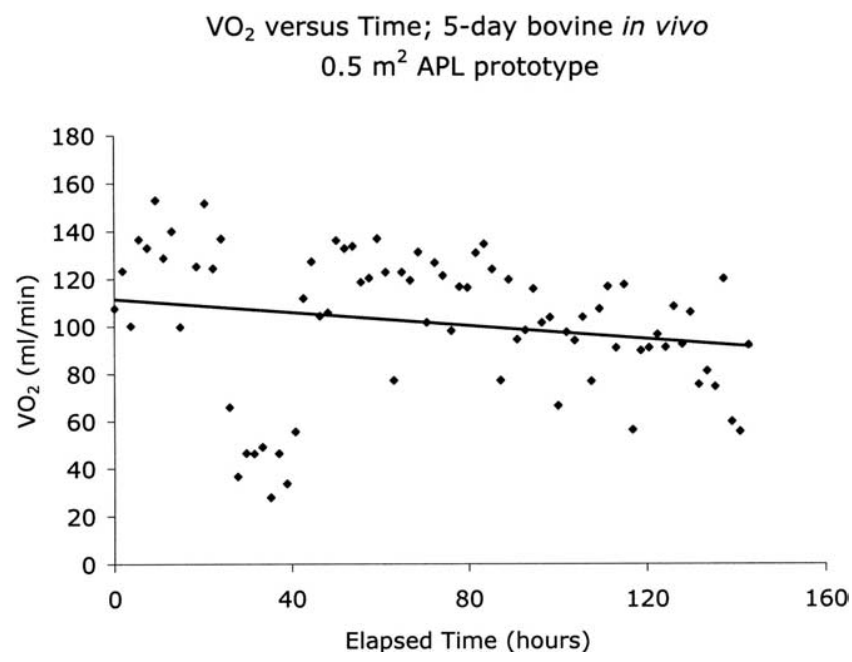

Figure 6. Oxygen transfer performance of the active mixing pump-lung device with a silicone-coated hollow-fiber membrane during a 5-day in vivo bovine evaluation. $\mathrm{VO}_{2}$, Oxygen transfer rate; $A P L$, active mixing pump-lung.

be a solution for these patients; however, these implantable devices are still in the development stage.

The group at the University of Michigan has led the field and favors a passive approach that includes the creation of transverse mixing or cross-flow to arrange the hollow-fiber membranes in a direction near perpendicular to the blood flow. ${ }^{15,16}$ This group has stressed the development of a low-resistance oxygenator that can be placed in a series with the native lung (PA-PA connection) without exceeding the ability of the right ventricle (RV) to manage flow through both the artificial and natural lungs. Although the passive designs improved gas transfer, this was only modest and required the continued use of a large surface area and complicated flow path and long exposure times that limited prospects for biocompatibility.

To reduce the surface area, active mixing techniques have been proposed to disrupt the formation of a boundary layer, thus reducing the diffusion resistance to $\mathrm{O}_{2}$ and $\mathrm{CO}_{2}$ in the blood phase and increasing the gas exchange efficiency. Active mixing techniques involve the introduction of active secondary flows to disrupt the boundary layer effect. Several active mixing designs have been proposed in the past, including rotating disk, inner cylinder rotating axial flow, and pulsed flow vortex shedding. Active mixing techniques have been shown to cause as much as a 5-fold increase in the rate of gas exchange. ${ }^{17-21} \mathrm{We}$ based our design on the concept of active convective mixing oxygenation within a constrained vortex. Our APL exhibited gas exchange performance far superior to that of commercially available devices. The pressure-flow curves of our APL indicate that the APL device is an acceptable pump for 
generating the normal adult cardiac output against a wide range of pressures. Our device, however, as an extracorporeal lung, can be sealed to permit the advantage of active mixing to translate into achievement of total body needs.

The evaluation of PFH generation and platelet and WBC preservation is promising. Clearly this device operates with a shear force, but transit time is very brief, which limits the adverse effects. The hemolytic performance of our device is comparable with that of acceptable blood pumps and commercial membrane oxygenators. It was observed that no thrombus deposition was found within the device during posttest examination. Our initial in vivo experiment showed that the APL device generated $\mathrm{O}_{2}$ transfer rates of approximately $175 \mathrm{~mL} / \mathrm{min}$ during 1-day acute studies and approximately $110 \mathrm{~mL} / \mathrm{min}$ during a 5-day study. Even the gas transfer rate during the 5-day study has not reached the level we like to have. However, the results with hemolysis and biocompatibility are promising. We are pleased with the initial performance and note that the functions of gas exchange and pumping are combined in the process. We have met our initial goal of gas transfer and pumping at physiologic rates. This permits us to disregard the need for a functional RV. For the APL to function for 21 days, it must incorporate a hollow fiber that is plasma resistant and has a minimal effect on the gas transfer performance, which is our continued effort.

\section{Conclusion}

Presently, a long-term intracorporeal artificial lung is still a dream. Progress to date by others and ourselves suggests a high likelihood of success for extracorporeal shorter-term lungs that can be switched in and out like dialysis devices. Our device is unique in that it incorporates an integrated pumping and active mixing principle for excellent gas transfer. To us, the ability to eliminate concerns about the native RV's ability to power blood through the lung is a major advantage. Certainly we acknowledge the risk of the combined function should there be a loss of disk spin. On the basis of our long experience with blood pumps, we believe that the risk of failure is low and, importantly, that our design does not result in hemolysis or loss of platelet and WBC counts when the patient undergoes an operation at physiologic rates of flow. We are encouraged by our pilot work and continue to iterate the design with consideration of function and biocompatibility.

\section{References}

1. Lick SD, Zwischenberger JB, Alpard SK, Witt SA, Deyo DM, Merz SI. Development of an ambulatory artificial lung in an ovine survival model. ASAIO J. 2001;47:486-91.
2. Zwischenberger JB, Wang D, Lick SD, Deyo DJ, Alpard SK, Chambers SD. The paracorporeal artificial lung improves 5-day outcomes from lethal smoke/burn-induced acute respiratory distress syndrome in sheep. Ann Thorac Surg. 2002;74:1011-6.

3. Wu ZJ, Antaki JF, Burgreen GW, Thomas D, Butler KC, Griffith BP. Fluid dynamic characterization of operating conditions for continuous flow blood pumps. ASAIO J. 1999;45:442-9.

4. Association for the Advancement of Medical Instrumentation. Cardiovascular implants and artificial organs-blood gas exchangers (oxygenators). Standard 7199. Association for the Advancement of Medical Instrumentation, Arlington (VA). 1996.

5. Malinauskas R. Plasma hemoglobin measurement techniques for the in-vitro evaluation of blood damage caused by medical device. Artif Organs. 1997;21:1255-67.

6. Steines D, Westphal D, Gobel C, Ruel, Rau G. Platelet function and hemolysis in centrifugal pumps: in-vitro investigation. Int $J$ Artif Organs. 1999;22:559-65.

7. Kawahito S, Maeda T, Yoshikawa M, Takano T, Nonaka K, Linneweber J, et al. Blood trauma induced by clinically accepted oxygenators. ASAIO J. 2001;47:492-5.

8. http://www.lungusa.org/research/data\&statistics (accessed on October 20, 2004).

9. Demling RH. The modern version of adult respiratory distress syndrome. Annu Rev Med. 1995;46:193-202.

10. http://www.ards.org/learnaboutards/whatisards/faq/faq3.html (accessed on September 26, 2004).

11. Rouby JJ, Lherm T, Martin de Lassale E, Poete P, Bodin L, Finet JF, et al. Histologic aspects of pulmonary barotrauma in critically ill patients with acute respiratory failure. Intensive Care Med. 1993;19: 383-9.

12. Grubitzsch H, Beholz S, Wollert HG, Eckel L. Pumpless arteriovenous extracorporeal lung assist: what is its role? Perfusion. 2000;15:237-42.

13. Liebold A, Philipp A, Kaiser M, Merk J, Schmid FX, Birnbaum DE. Pumpless extracorporeal lung assist using an arterio-venous shunt. Applications and limitations. Minerva Anestesiol. 2002;68:387-91.

14. 2003 Annual Report of the US Organ Procurement and Transplantation Network and the Scientific Registry of Transplant Recipients: Transplant Data 1993-2002. Department of Health and Human Services, Health Resources and Services Administration, Office of Special Programs, Division of Transplantation, Rockville (MD); United Network for Organ Sharing, Richmond (VA); University Renal Research and Education Association, Ann Arbor (MI).

15. Chambers SD, Merz SI, Mcgillicuddy JW, Bartlett RH: Development of the MC3 Biolung. In IEEE, Proceedings of the Second Joint EMBS/BMES Conference, Annual Fall Meeting of the Biomedical Engineering Society, Houston (TX), October 2002: p. 1581-1582, Omnipress, Madison (WI).

16. Lynch WR, Montoya JP, Brant DO, Schreiner RJ, Iannettoni MD, Bartlett RH. Hemodynamic effect of a low-resistance artificial lung in series with the native lungs of sheep. Ann Thorac Surg. 2000;69:351-6.

17. Gaylor JD, Smeby LC. The Taylor-Vortex membrane oxygenator: design analysis based on a predictive correlation for oxygen transfer. In: Dawids SD, Engell HC, editors. Physiological and clinical aspects of oxygenator design. Luxembourg: Elsevier/North-Holland Biomedical Press; 1976.

18. Hattler BG, Federspiel WJ. The artificial lung. In: Banner NR, Polak JM, Yacoub MH, editors. Lung transplantation. Cambridge (UK): Cambridge University Press; 2003.

19. Makarewicz AJ, Mockros LF, Anderson RW. A dynamic intravascular artificial lung. ASAIO J. 1994;40:M747-50.

20. Makarewicz AJ, Mockros LF, Anderson RW. A pumping intravascular artificial lung with active mixing. ASAIO J. 1993;39:M466-9.

21. Makarewicz AJ, Mavroudis L, Mockros LF. New design for a pumping artificial lung [abstract]. ASAIO J. 1996;42:70. 